\title{
O SENTIDO DAS INSTITUICÕ̃ES ESCOLARES NA PROFISSÃO DOCENTE DESDE A PERSPECTIVA BIOGRÁFICA
}

\author{
Rosa María Torres Hernandez*
}

RESUMO: $O$ artigo analisa, a partir das autobiografias, os significados que mobilizam os docentes de educação básica em seu encontro com as instituições escolares. Aborda como nestas instituições se estabelecem referências identificadoras substantivas para a profissão docente, para isso se interpretam as narrações autobiográficas levando em conta: 1) as constantes na "variação da velocidade" no discurso, 2) os trajetos, 3) as tensões e contradições. $\mathrm{O}$ estudo das autobiografias mostra que a experiência na instituição de educação primária é substantiva para a profissão docente. O normal é o lugar da identidade profissional, mas em articulação com a experiência acumulada na escola primária, não somente pelos vínculos e sim, além disso, graças às trajetórias e as contradições sustentadas no estabelecimento escolar.

Palavras-chave: Autobiografía; Docentes; Formação; Instituições.

\section{LO SENTIDO DE LAS INSTITUICIONES ESCOLARES EN LA PREFESIÓN DOCENTE \\ A PARTIR DE LA PERSCPECTIVA BIOGRÁFICA}

RESUMEN: El artículo analiza, a partir de las autobiografías, los significados que movilizan los docentes de educación básica en su encuentro con las instituciones escolares. Aborda cómo en éstas instituciones se establecen referencias identificadoras sustantivas para la profesión docente, para ello se interpretan las narración autobiográfica tomando en cuenta: 1) las constantes en la "variación de la velocidad" en el discurso, 2) los trayectos, 3) las tensiones y contradicciones. El estudio de las autobiografías muestra que la experiencia en la institución de educación primaria es sustantiva para la profesión docente. La normal es el lugar de identidad profesional pero en articulación con la experiencia atesorada en la escuela primaria, no sólo por los vínculos sino, por lo demás, gracias a las trayectorias y las contradicciones sostenidas en el establecimiento escolar.

Palabras-clave: Autobiografía; Docentes; Formación; Instituciones.

* Doctora en Pedagogía en la Universidad Autónoma de México (UNAM). E-mail: rrmth2000@gmail.com 


\section{THE MEANING OF THE SCHOOL' INSTITUTIONS IN THE}

\section{TEACHING PROFESSION BASED ON BIOGRAPHICAL PERSPECTIVE}

ABSTRACT: The article discusses, on an autobiography basis, the meanings, which activate basic education teachers, in their encounters with educational institutions. It addresses how these institutions are established in identifying substantive references to the teaching profession. In order to do that; it interprets the autobiographical narrative, taking into account the next: 1) the constant in the variation of speed "in the speech, 2) the paths, or the Right track 3 ) the tensions and contradictions. The study of the autobiographies clearly show that experience or expertise within the institution of education is substantial for the teaching profession. The School for Educators and the teaching Profession (Escuela Normal Superior) is the place of professional identity, due to its recognized name and gained experience, it is treasured by all elementary schools, not only for the links and connections it has but, moreover, through the paths and contradictions held on campus to establish the school system. Keywords: Autobiography; Teachers; Training; Institution.

\section{INTRODUCCIÓN}

Desde el año 2001 imparto un seminario en el que se analiza la perspectiva biográfica en la investigación educativa, los asistentes son estudiantes interesados en discutir ese tópico para construir su tesis, empero también, porque son docentes universitarios o normalistas preocupados por apropiarse de su historia personal para trabar "un diálogo" con su formación como profesores. En el seminario, los docentes construyen su autobiografía, con ello hacen un relato frente a otros y plantean las vicisitudes de su formación, la enfrentan como tarea social y se hallan con un mundo en el que reconocen que otros experimentaron dificultades y logros de manera similar a ellos.

En el espacio que brinda el seminario la escritura es la piedra de toque, los docentes redactan su autobiografía y la comparten, a diferencia del lugar de la nada (por ejemplo, el autismo o la anorexia) la escritura es una forma de tiempo perdido y tiempo recobrado que, no por ello niega su fragmentación. Las palabras se hacen más que signos y aparecen como convicciones y fetiches que cristalizan en una sensibilidad dramática por fin capturada, acomodada, apaciguada; porque el narrador, en este caso el docente, es una pasión que puede decirse, la narración actúa a modo de comprensión en el giro narrativo.

El seminario me permite tener un corpus ${ }^{1}$ de escritos autobiográficos para estudiar la formación de los profesores y su profesión. La for- 
mación, en su sentido amplio, es dar forma, darse forma, es "la dinámica de un desarrollo personal" (FERRY, 1990). Si la formación es comprendida así, entonces se hacen a un lado dos ideas: la primera tiene que ver con la fantasía Pigmalión, donde se cree que una persona puede modelar a otra. La segunda, está relacionada con la fantasía del Ave Fénix, que se consume y renace de las cenizas, preservando la idea del individuo que puede formarse a sí mismo. Considerar a la formación como dinámica de desarrollo personal y social me permite tomar la siguiente premisa: nuestros recuerdos se relacionan con todo aquellos que nos "da forma", esos recuerdos pueden aparecer como experiencias fuertes y cruciales, pero también en el curso de largos y continuos aprendizajes en la experiencia (BEILLEROT, 1998).

En el proceso de "darse forma" el profesor constituye social y psíquicamente su vocación, los sujetos no se identifican con la tarea sino con una imagen fraccionaria de la institución docente, o también, se pueden identificar con los grupos más arcaicos en su experiencia como alumnos. Así, van hacia lo interno, se repliegan en una historia asentada en la novela personal e institucional.

En este trabajo analizaré los tipos de significados que los sujetos movilizan en su encuentro con las instituciones escolares, el punto es cómo en éstas establecen referencias identificadoras que pueden leerse en la narración a partir de la concordancia de la velocidad en la descripción que el docente realiza y la comprensión de las acciones de producción y recepción de los sentidos que el sujeto narra. La presentación del análisis la realizo en tres partes: 1) constantes en la "variación de la velocidad" en el discurso, 2) trayectos, 3) tensiones y contradicciones.

Las autobiografías son una vía hacia las experiencias y acciones de los profesores; la narrativa autobiográfica permite reconstruir acciones, pero también reconocer huecos de la formación en los momentos claves de la vida. Para analizar los textos narrativos es importante reconocer que el texto no es la acción, sólo la representa. En esa representación existen formas detalladas de lo que se hace y de las maneras de proceder para hacerlo; en particular interesan las acciones relacionadas con la vida en las instituciones escolares con claro propósito formal de enseñanza y aprendizaje.

Las instituciones son lugares de existencia para los sujetos, son terrenos expresivos de la práctica, los saberes, la permanencia y los discur- 
sos. Las instituciones dan sentido y constituyen un sistema de pensamiento. Asimismo, las instituciones son espacios de tensión entre la norma y el sujeto, entre lo que el sujeto piensa y lo que crea (instituye); esto es, la institución no es sólo una fase objetiva sino sobre todo responde a una cara imaginaria y simbólica de vínculos entre los sujetos (KÄES, 1998; CASTILHO, 2005; REMEDI, 2006). Ahora bien, en toda institución hay un intersticio, es decir, el sujeto trabaja con zonas de incertidumbre y por ello el uso de la norma sufre una dinámica a partir del sujeto (FERNÁNDEZ, 1994).

La escritura autobiográfica es, en el estudio de la formación, un "espacio potencial" de la profesión docente, algunas de las razones para verlo así son:

- Al parecer en la autobiografía el lector es indispensable, de hecho el lector realiza un papel activo de colaboración, en este sentido el texto autobiográfico considera al "testigo necesario".

- Según el enfoque pragmático de la lingüística, el lector se convierte en un receptor ideal, reflejo especular del autor, una especie de doble.

- Como propone Bajtín (2003), en la autobiografía se considera al autor, al personaje y al interlocutor como elementos constitutivos del texto. La entonación, el estilo y la forma misma del texto dependen de la relación y el grado de interacción que existe entre ellos.

- En el texto autobiográfico no está en juego la verdad o la ficción, sino el orden de verosimilitud; de tal suerte que queda liberado de ser verificable y lo separa de la utilidad inmediata.

- Los textos autobiográficos pertenecen a la "forma incompleta", es un escrito del estilo que se somete voluntariamente al cortocircuito, a las interrupciones y a los traspiés.

- La triple identificación autor $=$ narrador $=$ personaje mezcla elementos pertenecientes a esferas diferentes, perdiéndose la relación autor/creador con lo cual se da la posibilidad de una estetización voluntariamente infiel.

- Lo que la autobiografía aporta es, en todo caso, una "ilusión de referencia”, y en cuanto fenómeno literario está sometida pragmáticamente a un determinado modo de lectura y comprensión.

- La autobiografía como un punto de partida más que de llegada, es una búsqueda moral y estética, una interrogación en una tumba 
abierta. Como dice Fernández (2006) estamos siguiendo un hilo de Ariadna que no sabemos hacia dónde nos conduce.

\section{Constantes en la "variación de la velocidad" en el discurso}

La escritura autobiográfica de los profesores es una labor que produce una narración, esta se encuentra entre la indeterminación de los hechos y la configuración de una trama; de tal suerte que la unidad y la coherencia de la vida que se escribe no es un efecto de sentido, sino más bien, un proyecto siempre previo a la dotación de sentido. Escritas las autobiografías se convierte en “... un discurso en su forma textual en la que se ponen en relación distintos componentes que se articulan con una determinada coherencia" (ZECCHETTO, 2005, p. 156).

La autobiografía es un discurso que se asienta en la escritura, la fijación en la escritura constituye al texto, en el planeamiento de Ricœur (2002) “... la fijación de la escritura se produce en el lugar mismo del habla, es decir, en el lugar donde el habla habría podido aparecer (...) lo que llega a la escritura es el discurso en tanto intención de decir, la escritura es una inscripción directa de la intención, aun cuando, histórica y psicológicamente, la escritura comenzó por transcribir gráficamente los signos del habla. La liberación de la escritura que la pone en el lugar del habla es el acto del nacimiento del texto" (p. 128-129).

El cronotropo de la escritura narrativa (BAJTÍN, 2003) se encuadra en la cultura, en la sociedad, en lo Otro y en los otros; se estable en el juego temporal-espacial, por esa razón recurro para el análisis a la semiótica donde la segmentación y secuencia se toman en cuenta para estudiar los "eventos" y los "hechos" porque necesariamente responden a coordenadas espacio-temporales.

Si bien, cuando hablo de la temporalidad en la narración autobiográfica discurro que el tiempo es un hecho de la conciencia y la memoria, ya que no puede menos que inscribirse en él, del mismo modo, considero sin reservas lo que Freud afirma, las huellas duraderas de excitación permanecen inconscientes, lo dicho conduce a la huella némica, es decir, no puedo obviar la pre elaboración y la liquidación de la transferencia, tal cual lo propone Kristeva (2001), o en términos de Green (2001), la heterocronía y al "recordar-repetir y reelaborar"; de eso también están consti- 
tuidas las narraciones autobiográficas. El reto frente al tiempo fragmentado es, que de cara a la existencia de historias en el interior de la esfera del individuo, de la cultura, de la especie, que se imbrican, se mezclan y a veces se oponen, viviendo cada una a su ritmo propio, según su tiempo no se reduce a las huellas visibles que deja (accesibles a la conciencia) ni aquello cuyo recuerdo es conservado por los traumas, no es sólo lo manifiesto sino de igual forma emerge lo latente.

No obstante, en la labor de investigación para acceder al tiempo fragmentado es necesario recurrir al modo manifiesto de la significación, es decir, a los términos que forman parte del discurso, para ello, apelo a la dinámica que el propio texto presenta. Así, el número de párrafos escritos para describir determinadas acciones y eventos plantea el orden de importancia que adquieren para el sujeto las instituciones escolares, esta forma de análisis la recupero del trabajo de Dominicè (2000), quien a su vez retoma la propuesta de Greimas (1983) de la semiótica narrativa que intenta explicar cómo se producen y cómo se capta el sentido de una narración.

En este momento me encuentro en el lugar de lo que Ricœur (2002; 2003) caracteriza como el momento de la explicación para intentar otro movimiento que es la compresión, voy a dibujar como trabajo el momento explicativo en las autobiografías, para acercarme a la complejidad de la escritura del otro e interpretarla. Tengo interés en ver las constantes en la formación de profesores normalistas para comprender aquello que permanece al servicio de la experiencia de la profesión docente, si bien, lo hago bajo la consideración de que trabajar sobre constantes en un corpus no desplaza la necesaria particularidad de la narración personal.

La "variación en velocidad" que le imprime a su texto un docente, permite ver el valor que tienen las diversas instituciones escolares su la vida, la variación la establezco a partir del número de párrafos dedicados, en la escritura, a narrar las experiencias y aconteceres en una institución educativa. Tal como lo indica Dominicè es ahí donde se abre la pregunta: ¿por qué tiene tanto cuidado los docentes en contar lo que hicieron en esas instituciones?

Así se observa en el caso de Cristina, maestra normalista, que cursaba el doctorado cuando escribió su autobiografía. 
Gráfica 1. "Variación de velocidad"

del discurso de Cristina acerca de las instituciones escolares

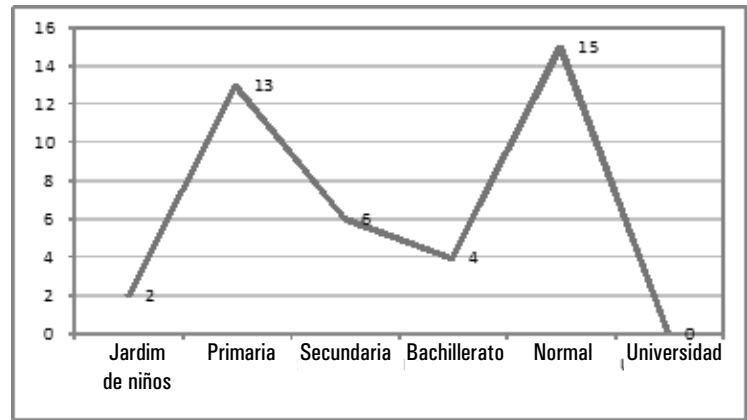

Me aboco a establecer las constantes en la "variación de velocidad" en la narración de ocho docentes normalistas.

Gráfica 2. "Variaciones en la velocidad" del discurso de ocho autobiografías con respecto a las instituciones escolares

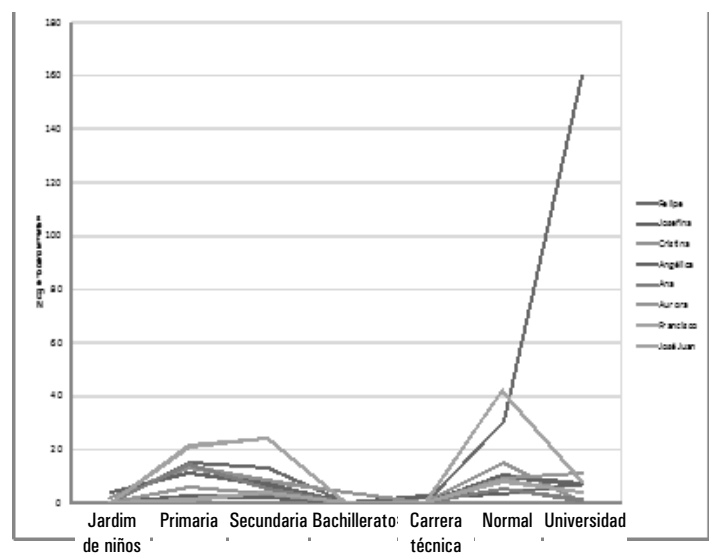

\section{Trayectos}

Si se toma en cuenta la "variación de la velocidad" en las autobiografías analizadas, el significado de la escuela primaria en la vida de los maestros normalistas aflora como determinante, de igual forma lo son sus años en la normal. La secundaria es una institución de segundo nivel de relevación y la universidad surge a modo de horizonte o proyección en la vida de los profesores. 
Además de la "variación de velocidad", el análisis de la formación de las ocho autobiografías lo hice, a partir de un esquema de tripartición -propuesto por Demetrio (1999) para la construcción de la autobiografía- que permite una reordenación de las tensiones existenciales, las inscripciones sociales y los develamientos psíquicos narradas en el texto autobiográfico. El esquema consta de tres partes: el incipit, la ruit y el exit. El incipit, comienzo, principio o inicio (sensorial y escénico-figurativos); la ruit el itinerario, y el exit como la conclusión son los componentes del esquema de tripartición.

En el caso de José Juan, se presenta una "variación de velocidad" mayor en la secundaria que en la primaria, por esto resulta interesante tomarlo como un caso particular para el análisis de tripartición puede producir otros sentidos para el análisis. El incipit en la primaria en la autobiografía de José Juan aparece de la siguiente forma:

En la escuela yo alcancé al igual que Lily y Mario [sus hermanos] a ir a la mejor escuela de la ciudad, la de los "riquillos", aunque no lo éramos, Mamá insistió a llevarnos a la Escuela Anexa a la Normal, Primaria "Vivanco". Donde estaban los mejores maestros, por las exigencias de la Normal y porque ahí practicaban los estudiantes. Estar en la anexa como maestro era el gran estatus, era ser los mejores maestros del pueblo.

\section{El itinerario o ruit uno acontece así:}

El rigor y la disciplina (militar) en la escuela era castrante... íbamos mañana y tarde. En primer año me topé con el profesor más autoritario y pedante que he conocido. Gracias a él cuando llegué a ser maestro jamás he sido autoritario ni arbitrario con mis alumnos, y gracias a él he sido un maestro crítico. Por ser un alumno de la anexa, creo que me hice desobediente y retador de la autoridad incompetente e incomprensiva. Sufrí horrores con ese maestro. Todos mis hermanos eran super estrellas al paso por la primaria y yo era la oveja negra, juguetón, desobediente, mala letra, descuidado en mis libros y libretas, no aprendí a leer, ni ortografía. Pero era muy listillo. Finalmente me pasaron a $2^{\circ}$, haber si mejoraba, no me reprobó Martín Guadalupe Elizondo, el super maestro de Sabinas. En $2^{\circ}$ año me tocó la maestra más dulce de la escuela, la maestra Lucybeny, me convertí en su amor, "el muñeco" me puso apodo que todavía en Normal algunos 'gachos' me lo gritaban. Pues con el cariño mejoré y me sentí más seguro y me fue mejor. Pero seguía batallando para leer y escribir. Creo que tengo alguna deficiencia, porque hasta ahora sé que se me cruzan las letras al leer (tengo un tipo de dislexia) que jamás me detectaron y que aún no sé que sea. 
Así narra la ruit dos José Juan:

Recuerdo muy bien que a los maestros y maestras de grupo, llevaban las clases bien planeadas y con mucho material, cuando tenían observación de los alumnos de la Normal, llenaban el salón especial, uno muy grande donde más de 30 alumnos normalistas bien encorbatados se sentaban alrededor del salón y empezaba la clase práctica el maestro titula.

Para entonces todos ya estábamos bien advertidos por el maestro de... cuidadito con el que lo hiciera quedar mal. Nos daba todas las indicaciones y reglas del juego. Como sentarnos, como sacar los libros, como abrirlos, como ponernos de pie por tiempos 1, 2, 3... igual al sentarnos, como formarnos, hasta la manera de cómo indicar cuando el o ella preguntara en clase... y muchas cosas más.

En la ruit tres dice el autor:

En sí la escuela era muy exigente y autoritaria. En tercero me tocó una maestra muy buena, de buen corazón y trabajé muy a gusto, aún con mis deficiencias y bajas calificaciones (por juguetón y distraído). Desde primero me había quedado un gran temor por el no aprender rápido, el cual me duró hasta la Normal.

En el exit José Juan escribe.

Aprender con temor fue mi mayor desdicha en la anexa, aunque el juego y los amigos mitigaron esa pena.

Al fin llegó la graduación... incrédulo porque al fin terminaba la primaria, gran fiesta de graduación para 1970-71. La tristeza porque la generación se separaría aunque todos iríamos a la misma secundaria. Casi nadie íbamos a tocar juntos, porque todas las primarias llegaban a la secundaria... la única en la ciudad: "Profesor Antonio Solís".

Quien Iba a pensar que la oveja negra de la casa iba ser el que más estudios lograra y que aún ande y se me haya hecho un opio el seguir estudiando y leyendo.

Algunos de los elementos expuestos en el texto de José Juan con respecto a la primaria se pueden ubicar en un eje de sucesión, esto es, el mundo en el que el tiempo de nuestro conocimiento consciente es de lo anterior y lo posterior, si bien esos pasos que en apariencia son secuenciales están flanqueados por el horizonte o contorno en un campo que "flota" entre el recuerdo y el olvido y donde extraemos la vivencia espacio-tiempo. 
Eje de sucesión

[incipit] La mejor escuela à $\rightarrow$ [ruit 1] rigor y disciplina castrante à $\rightarrow$ [ruit 2] cuidadito con hacer algo mal à $\rightarrow$ [ruit 3] me tocó una maestra buena $y$ de gran corazónà $\rightarrow$ [exit] llegar a la única secundaria de la ciudad.

Además del eje de sucesión existe el eje de intención, ese fluir continuo de deseos, pasiones, metas, intenciones, que se podría llamar el presente móvil, un presente que se intuye en movimiento desde el pasado hacia el futuro, por la intención es que creemos que el tiempo posee una dirección; ésta expresa el estar dirigido a metas, cualidad de la conducta intencional que se describe en la autobiografía como una historia de múltiples aristas.

Eje de intención

[incipit] Ir a la mejor escuela de la ciudad, la de los riquillos à $\rightarrow$ [ruit 1] tope con maestro pedante, gracia a él he sido un maestro crítico à la oveja negra à el muñeco àun tipo de dislexia à $\rightarrow$ [ruit 2] observación de los alumnos de la normal à $\rightarrow$ [ruit 3] trabajé con mis deficiencias y bajas calificaciones à $\rightarrow$ [exit] aprender con temor fue mi mayor desdicha en la anexa, aunque el juego y los amigos mitigaron esa pena.

El análisis de los ejes de sucesión y de intención lo llevé a cabo con las ocho autobiografías, porque esos inicios, rutas y conclusiones que narran los docentes en los establecimientos escolares de la primaria y la secundaria, marcan las formas de apertura, itinerario y salida en la institución de formación profesional, que en este caso es la Normal de maestros. En la narración de José Juan la continuidad es evidente (no es así en todos los casos). El incipit en la normal lo describe así:

Pues la Normal era un hervidero de gente el día del examen, de nuevo a volvía a la que había sido mi escuela primaria, los recuerdos pasaron por mi mente de todos los años de mi niñez, hoy me sentía adolescente. Retornaron a mí aquellos días felices y aquellos días de inseguridad y miedo.

Ahora, iba a enfrentar la barrera de estudiar la Normal, y pertenecer a la escuela de arriba, ya que la Normal estaba en la planta alta de la escuela primaria Anexa a la Normal. Legó el día, más de 350 aspirantes de Sabinas, Villaldama, Bustamante, Anáhuac, Lampazos, Garza Ayala, Vallecillo, Agualeguas y otros pueblitos, ciudades, ranchería del norte de Estado. 
El análisis hace pensar en la existencia de una especie de continuidad marcada entre la escuela primaria y la formación como maestro normalista, no porque las otras instituciones escolares no tengan importancia, pero el significado de la narración recae en estas dos entidades escolares, de hecho para efectos de lo que me ocupa, puedo afirmar que la profesión docente en el magisterio es la depositaria de la experiencia en la escuela primaria. De la misma manera, las narraciones permiten discurrir que, las instituciones escolares son lugares donde se encuentran los trayectos de los sujetos con los "ciclos metabólicos" de establecimiento escolar, esos ciclos crea formas de incluir o reclutar, triturar y devolver a la persona (GOFFMAN, 1991) y encuentran tierra fertíl en aquellos que buscan rutas, intersticios, componendas, etcétera, para cubrir el ciclo metabólico de los establecimientos escolares. Goffman (1991), dice:

Yo creo que en biología (aunque no sé gran cosa), un organismo traga algo, lo utiliza y, después, lo expele bajo la form de desecho, menos complejo que el producto total. Sin embargo, enla vida social, la materia humana de ciertas instituciones se reinyecta a menudo estratégicamente en la sociedad, donde van a representar un papel importante. En resumen, la organización encuentra su finalidad en el papel que despés representarán fuera los desechos (p. 110-111).

\section{Tensiones y contradicciones}

Las tensiones y contradicciones son elementos para entender la articulación de sentido en las porque identifica los retos de transformación que los profesores advierten. En la vida de José Juan las formas identificación tanto por acción contraria a su opositor "el maestro autoritario" como por introyección de "la maestra más dulce" dieron movimiento a la transformación casi épica de las formas contradictorias de la experiencia en la escuela primaria, veamos cuáles eran esas contradicciones: 
Figura 1. Cuadro semiótico de la escuela primaria José Juan

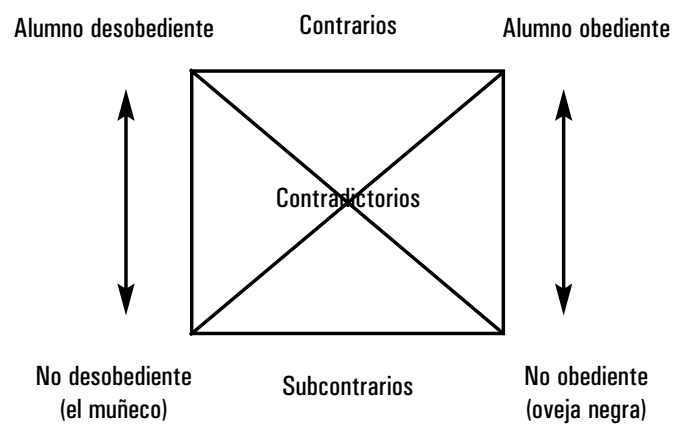

Las contradicciones y subcontradicciones pueden coexistir, pero las diagonales no; y las verticales como líneas de implicación son necesarias para el encuentro con la historia de la institución, así como, para las formas de hacer del sujeto en la institución.

El análisis de las contradicciones me permite reflexionar en la importancia de los vínculos y relaciones de los sujetos en la escuela primaria que reflotan en los subcontrarios aún con la existencia, quizá necesaria, de los contrarios para establecer un juego de transformaciones más que de destino, es decir establecer rutas del devenir profesional. Es claro que los vínculos no serían los únicos elementos a considerar pero no son cosa menor, hablan de la importancia para la profesión docente de los propios profesores y de la escuela como un lugar de concentración experiencia con potencia inhibidora y transformadora de futuro.

Cuentan también las rutas, las formas de afrontar las contradicciones, los mecanismos de sobrevivencia y las alianzas estratégicas con la novela institucional. José Juan es participe de la esa novela, es constantemente reclamado por el mandato social al que responde el establecimiento "ser la mejor escuela de la ciudad"; no podía ser menos que eso, era la escuela anexa a la normal, era parte del hacer cotidiano de los normalistas en formación.

Iré ahora a los contrarios sostenidos por José Juan en la Normal, él estudia la preparatoria y la Normal al mismo tiempo, lo que le permitirá entrar a la licenciatura en historia, tiene rabia porque no se puede negar- 
se al mandato familiar de seguir la carrera magisterial pero no puede oponerse, además dice:

Pues, esa filosofía de alguna u otra manera convencía a todos los adolescentes. Hombres y mujeres han de ser diferentes a los universitarios, la universidad era otro estrato social, medio alto y alto. En el pueblo eso nos enganchó a la mayoría, era el despertar al deber social, toda la energía juvenil se encaminaba al saber, al deporte, a la artística, al baile, a la excursión, más allá de la pérdida de tiempo ocioso que se veía en los universitarios. Nosotros no podíamos ser fresas.

Pero mis amigos de la secundaria seguían frecuentándose y todos estaban en la prepa e iban a seguir una carrera universitaria, me seguían aceptando porque también era universitario-nocturno. El lenguaje, el pensamiento y la actuación de esta raza no pasaba por el deber ser, ni los héroes, ni la clase humilde, ellos eran distintos, eran puro juego, poca responsabilidad social, enfrentaban las disciplinas duras del saber física, química, matemáticas, álgebra, contaduría, aritmética, etc. La discusión era saber los sabores científicos, no sociales, ni de lucha, eran más libros en su pensar y actuar.

Yo en el fondo no lo era, ya estaba atravesado por la responsabilidad social de clase humilde y luchadora que la Normal inyectaba sin darnos cuenta. Renegaba de las estupideces de ésta raza, pero también reconocía que ellos la pasaban más libres de ataduras sociales, eran más plenos en su adolescencia, uno finalmente se detenía en ciertas actuaciones.

Figura 2. Cuadro semiótico escuela Normal José Juan
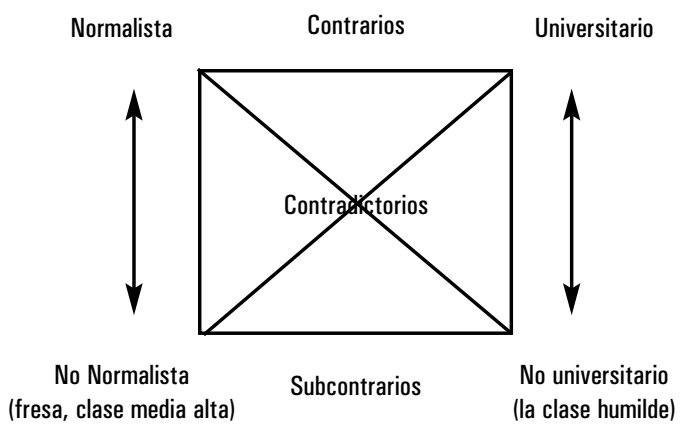

Cabe decir que los sujetos se incluyen en las profesiones no sólo por el buen desempeño académico o la motivación personal, se debe tomar en cuenta el hecho de que en las instituciones se establecen acuerdos sociales y formaciones psíquicas intermedias que tiene un “... carác- 
ter bifronte de la reciprocidad que induce en los elementos que las ligan, la comunidad que se consolida mediante pactos, contratos y consensos inconscientes, articulan de esa manera las relaciones del elemento con el conjunto en figuras diversas: de ensamble, de inclusión mutua, de co-inherencia o de inversión continua (según el modelo de la banda de Moebius) (KÄES, 1998, p. 33).

Tal como lo señala José Juan existen ritos necesarios y formas diversas de inclusión que articulan la historia del sujeto y la historia de la institución, esto a su vez marca formas de ser y hacer en la docencia.

A eso conduce la escritura de José Juan:

Cada año salíamos a las calles a desfilar vestidos de revolucionarios con machetes, carabinas, caballos y burros por las calles del pueblo. Éramos realmente mexicanos convencidos de nuestros héroes, era un gran espectáculo el que la Normal organizaba cada año. La banda de guerra, era otro rito, cada día festivo toda la banda dormía en la Normal y a las 5 de la mañana iniciaban a tocar por horas hasta izar la bandera. Era toda una formación cívica y patriótica del deber ser, y la lucha contra la pobreza.

Todos los maestros y maestras del personal tenían esa mística, trabajo y más trabajo para salir adelante, nada de "chiflazones" esto no era para la clase humilde.

Todos esos años transcurrieron entre trabajo, estudio, responsabilidad, diversión sana, deporte y compañerismo, fenómeno generalizado en las generaciones.

Se daba una situación rara, en los bailes, bodas de fin de semana, todos los normalistas nos saludábamos como hermanos, había una identificación de clase, los grupos de amigos eran generalmente de puros normalistas. Yo en lo personal tenía que convivir con amigos normalistas con los cuales convivía y los cuales eran solidarios en cualquier bronca que se te presentara, como es clásico en los pueblos.

Asimismo, la normal y la primaria se vinculan por el juego de contrarios que se establece en la vida de José Juan, he aquí un anudamiento de sentidos de alta complejidad pero de gran interés para la profesión. Así la subversión de alumno desobediente versus alumno obediente, así como, la subcontradicción "el muñeco" y la "oveja negra" se replantean en otro momento de la vida, en donde José Juan hace del normalismo una hermandad solidaria de grupo y clase, más allá del estereotipo personal y de las dificultades de aprendizaje sufridas en la escuela primaria. Quizá podríamos decir que la escuela Normal es subsidiaria de la escuela primaria en la profesión docente. 


\section{A modo de cierre}

El interés en la profesión docente me coloca ante la búsqueda de los sentidos de los sujetos pero también en la perspectiva de los acentos colectivos, bajo la premisa de que el relato autobiográfico por sí mismo es de gran riqueza, pero desde la investigación esa riqueza tiene que ser pensada con fines interpretativos, lo cual ni lo demerita, ni necesariamente le aporta.

Otro asunto que tomo como referente, es la profesión como social y cultural, por ello, a pesar de la premisa cada vida vale por sí misma, encuentro una fuente de reflexión al leer las narraciones autobiográficas como un discurso, donde más allá de los enfoques posibles, encuentro formas de articulación para comprender los significados; esos significados se revisan en el seminario con los docentes lo que incrementa en ellos el pensamiento crítico a través de una exploración permanente de sentido, hacen una interpretación hermanados con otros profesores, revisan su aprendizaje y aprecian los influjos identificatorios que experimentaron en los contextos de las instituciones escolares.

Presenté el análisis de incipit, ruit y exit de sólo uno de los discursos narrativos, pero el análisis de la "variación de la velocidad" en el discurso, establece ciertas constante en la autobiografías de los profesores normalistas. Sin olvidar que aparecen formas contradictorias en las acciones y tensiones en las instituciones escolares.

En el estudio de las autobiografías la experiencia acumulada muestra que la experiencia en la institución de educación primaria es sustantiva para la profesión docente, es en cierto sentido la puerta de Jano. La normal es el lugar de identidad profesional pero en articulación con la experiencia atesorada en la escuela primaria, no sólo por los vínculos sino, por lo demás, gracias a las trayectorias y las contradicciones sostenidas en el establecimiento escolar.

El sujeto es y se hace en la institución escolar en una dinámica que construye y reconstruye en un tejido de múltiples texturas que comprende la novela institucional y su relación con sus formaciones sociales, culturales y psíquicas. Así, se observa en las continuidades y las formaciones de sentido, puesto que éstas son la historia del individuo y la historia de la sociedad. Las historias dan idea sobre cómo se forman los profesores, qué hacen, qué los motiva para ser docentes y qué caminos recorren 
para lograrlo. Asimismo, la escritura de los profesores en las formas de la "literatura gris", que reivindica Allouch (2001), es decir todo aquellos que parece trivial y ordinario. No obstante, eso es lo que permite pasar de la chora semiótica al gusto vuelto visión gracias al estilo del autor, es la reflexión del sujeto de su estar en la vida, saberse estar en vida singularmente y a la vez -como dice Levinas (1997) - "vivir de buena sopa”, de aire, de luz, de espectáculos, de trabajos, de sueños como parte constitutiva de ser docente. 


\section{BIBLIOGRAFÍA}

ALLOUCH, Jean. Erótica del duelo en tiempo de la muerte seca. México: Psicoanalítica de la letra. 2001

ARFUCH, Leonor. El espacio biográfico. Dilemas de la subjetividad contemporánea. Buenos Aires: Fondo de Cultura Económica. 2002

BAJTÍN, Mijail. Estética de la creación verbal. México: Siglo Veintiuno. 2003.

BEILLEROT, Jacky. La formación de formadores. Buenos Aires: Facultad de Filososfía y Letras UBA, Novedades Educativas. 1998

CASTILHO, William, César. Movimiento institucionalista. Principiais escolas. En

CASTILHO W. C., Análise institucional na vida religiosa consagrada. Belo Horizonte: Publicaciones CRB. 2005. p. 59-90.

DEMETRIO, Duccio. Escribirse. La autobiografía como curación de uno mismo. Buenos Aires: Paidós. 1999

DEMINICÈ, Pierre. Learning from our lives: Using educational biography with adults. San Francisco: Jossey-Bass Inc. 2000

FERNÁNDEZ, Lidia. Instituciones educativas. Dinámica institucional en situaciones críticas. Buenos Aires: Paidós. 1994

FERNÁNDEZ, Lidia. Esapacios institucionales dela educación. Algunos componentes nucleares en la identidad institucional y sus consecuencias para el análisis. En LANDESMANN, Monique. Instituciones educativas. México: Juan Palos. 2006. p. 11-25.

FERRY, Gilles. El trayecto de la formación. México: Paidós. 1990

FRIGERIO, Graciela, y DIKER, Gabriela. La transmisión en las sociedades, las instituciones y los sujetos. Buenos Aires: Novedades Educativas. 2004

GOFFMAN, Erving. Los momentos y sus hombres. Barcelona: Paidós. 1991

GREEN, Andre. El tiempo fragmentado. Buenos Aires: Amorrortu. 2001

GREIMAS, Algidas Julien. La semiótica del texto. Buenos Aires: Paidós. 1983

GREIMAS, Algidas Julien. Semiótica estructural. Madrid: Gredos. 1987

KÄES, Rene. La institución y las instituciones. Buenos Aires: Paidós. 1998

KRISTEVA. Julia. El porvenir de la revuelta. México: Fondo de Cultura Económica. 1999

LEVINAS, Emmanuel. Fuera del sujeto. Madrid: Caparrós. 1997

REMEDI, Eduardo. Instituciones educativas. México: Plaza y Valdes. 2004

RICCEUR, Paul. Del texto a la acción. México: Fondo de Cultura Económica. 2002

RICCEUR, Paul. El conflicto de las interpretaciones. México: Fondo de Cultura Económica. 2003

ZECCHETTO, Victorino. Seis semiólogos en busca del lector. Buenos Aires: La Crujía. 2005 


\section{NOTA}

1 El corpus consta de 30 autobiografías, algunas de ellas escritas en dos partes. Para este trabajo tomaré sólo ocho de ellas. Los autores de las autobiografías dan su autorización en el seminario para que sus textos se usen con fines de investigación educativa.

Recebido: $12 / 03 / 2010$

Aprovado: $17 / 09 / 2010$

\section{Contato:}

Universidad Pedagógica Nacional

Carretera al Ajusco, 24

Col. Héroes de Padierna Del. Tlalpan

México - Distrito Federal

14200 